\title{
Tile Boundary Artifacts Reduction of JPEG2000 Compressed Images
}

\author{
Singara Singh ${ }^{1}$, R. K. Sharma ${ }^{2}$ and M. K. Sharma ${ }^{3}$ \\ ${ }^{1}$ Assistant Professor, ${ }^{2}$ Professor, ${ }^{3}$ Associate Professor \\ School of Mathematics and Computer Applications, \\ Thapar University, Patiala, Punjab, INDIA-147004
}

\{singara, rksharma, mksharma\} @thapar.edu

\begin{abstract}
:
This paper proposes a post-processing technique for reducing tile boundary artifacts which occur in an image when it is compressed at low bit rate using JPEG2000 standard. Symmetric extension and difference in quantization accuracy between the tiles of the image are the main factors behind the tile boundary artifacts in JPEG2000 compressed images. In this paper, we have analyzed the effect of quantization on the region of the tile boundaries of JPEG2000 compressed images. The analysis confirms that tiling artifacts are reduced by updating the high pass reconstructed samples lying on the boundary of the image tiles where the artifacts occur. The post-processing is applied on the output of JPEG2000 coding system and thus it can easily be blended with JPEG2000 standard.
\end{abstract}

\section{Keywords:}

JPEG2000, MSE, PSNR, DWT, boundary artifacts, quantization.

\section{Introduction}

JPEG2000 is the state-of-art image and video compression standard developed jointly by the International Organization for Standardization (ISO) and the International Electrotechnical Commission (IEC) [1-6]. It offers improved compression performance for various type of images including natural, binary, remote sensing, scientific and medical images. This standard primarily, uses Discrete Wavelet Transform ( $D W T$ ), followed by scalar quantization (as required) and the entropy coding. In JPEG2000 coding system, when an image is larger than the available memory for elaboration, the image is divided into tiles. Tiles are rectangular regions of the image that are transformed and encoded independently. Tiles can be of any size, and it is also possible to consider the whole image as a single tile. Once the size of the tile is chosen, all the tiles will have to have the same size. The main advantage of dividing the image into tiles is that the decoder will need less memory to decompress the image and it can opt to decode only selected tiles to achieve a partial decoding of the image. As such, tiling can give better memory utilization and better

Natarajan Meghanathan, et al. (Eds): ITCS, SIP, JSE-2012, CS \& IT 04, pp. 281-290, 2012.

(C) CS \& IT-CSCP 2012

DOI : $10.5121 /$ csit.2012.2126 
access under certain circumstances. It can significantly reduce the complexity on the encoder side. It is also useful in handling high resolution images. A difficulty that tiling introduces is the blocking artifacts that are significant when the images are compressed at low bit rate using JPEG2000 coding system. There are two major factors of the tiling artifacts in JPEG2000 images. One factor is the symmetric extension of the original image samples which is performed prior to applying the wavelet transform. Other factor is the difference in quantization accuracy which is due to difference in quantization errors in each tile.

A good number of researchers have worked on reducing the tile boundary artifacts of JPEG2000 compressed images. Berkner and Schwartz [7] proposed a method for reducing boundary artifacts using projection onto convex set in the encoder. Their method eliminates the blocking artifacts by computing approximations that match the smoothness of the compressed image. It has been noted that it is very difficult to apply pre-processing while implementing the encoder, in order to reduce tiling artifacts. Kharitonenko et al. [8] have proposed low complexity wavelet transform method which uses a point symmetric extension method in place of symmetric extension method of JPEG2000 standard, for improving the image quality. Their method deals efficiently with blocking artifacts without requiring any information from the neighboring tiles. In [9], the tile size was chosen to be odd so as to remove the tiling artifacts. The authors have here established that for the same compression rate, an image compressed using an odd tile length low pass first convention has less boundary artifacts than an image compressed using even tile sizes. In [10], a pre/post filtering approach has been proposed for JPEG2000 tiling artifacts removal. Here, a prefilter is applied at tile boundaries before wavelet decomposition, and a post filtering is applied at tile boundaries after wavelet reconstruction on the decoder side. The optimal bit allocation is derived and then corresponding boundary distortion for a given pre/post filter pair is calculated. The optimal pre/post filters are then obtained by minimizing the reconstruction errors of the boundary filter bank. Qin et al. [11] proposed a post-processing method to reduce tiling artifacts with the help of max-lift wavelet subband decomposition. They adaptively filtered the subband coefficients using soft thresholding. The post-processing is carried out after decompression of image by the JPEG2000 coding system. Hashimoto et al. [12] have also proposed a method for reducing tiling artifacts. Their method predicts the presence of tiling artifacts at a tile boundary in the rate control process and then improves quantization accuracy by using post quantization control locally. All these approaches are effective in reducing tiling artifacts of JPEG2000 compressed images but we need to reduce these tile boundary artifacts to even lower levels.

In this paper, it is shown that the tile boundary artifacts in JPEG2000 compressed images are due to larger errors of high pass boundary samples. The high pass reconstructed sample close to the tile boundaries have larger error than the error in the neighboring low pass reconstructed sample. We will show in the next section that by updating these high pass reconstructed samples with the help of neighboring samples, the tile boundary artifacts in the reconstructed image are reduced.

The rest of the paper is organized as follows. Section 2 describes the cause of boundary artifacts in tiles of JPEG2000 compressed images. Section 3 illustrates how these boundary artifacts can be reduced by post-processing of the boundary samples of the reconstructed JPEG2000 images. This section also contains the proposed method and the results of this study. Section 4 concludes the paper. 


\section{Tile Boundary Artifacts Analysis}

\subsection{Wavelet Transform}

Wavelet transform is a subband transform which transforms images from spatial domain to frequency domain. To achieve efficient lossy and lossless compression within a single standard, two wavelet transform filters are used in JPEG2000 standard. One is CDF 9/7 floating point filter which is used for lossy compression and the other is LeGall $5 / 3$ which is simple to implement and has lossless capability. Two dimensional $D W T$ is the combination of a one dimensional (1-D) horizontal and 1-D vertical $D W T$. The original image is decomposed to produce low pass and high pass filtered outputs of the image. These filtered outputs are then down-sampled by a factor of two in order to produce a set of subbands of the original image. Because of the down-sampling process, the total number of wavelet coefficients is same as the number of original image samples. The output of this process is the set of four subbands - LL (Low Low), $L H$ (Low High), $H L$ (High Low) and $H H$ (High High) subbands. This wavelet decomposition process can be applied iteratively on the $L L$ subband to get a multiresolution decomposition of the original image. $D W T$ decorrelates the input image and also provides a good energy compaction

To produce the reconstructed image from the compressed image, different filter pairs are needed. The subband samples of the image are first upsampled by a factor of two and then filtered to produce reconstructed low pass and high pass versions of the original image. The outputs of the reconstructed filters are then summed to produce the final reconstructed image.

To keep the number of subband samples same, as the number of original image samples, the original image is symmetrically extended about the boundaries before performing the wavelet transformation. The length of the extension depends upon the number of wavelet filter coefficients used in the wavelet transform. If the number of wavelet filter coefficients is $m$, then the length of extension is $\left\lfloor\frac{m}{2}\right\rfloor$ in both directions, where $\left\lfloor\frac{m}{2}\right\rfloor$ indicates the largest integer not exceeding $\frac{m}{2}$. In JPEG2000 image compression standard, a whole sample symmetric extension is used to extend the boundaries of the image/tile. For example, if the input image data is $x[0], x[1], \ldots, x[n-2], x[n-1]$, then a whole sample symmetric extension would give: $x[2], x[1], x[0], x[1], \ldots, x[n-2], x[n-1], x[n-2], x[n-3]$

This extension depends upon the number of wavelet filter coefficients used in wavelet transform. If the number of wavelet filter coefficients is $m$, then the length of extension is $\left\lfloor\frac{m}{2}\right\rfloor$ in both directions, where $\left\lfloor\frac{m}{2}\right\rfloor$ indicates the largest integer not exceeding $\frac{m}{2}$.

Focus of this paper is to analyze and reduce the boundary artifacts that occur at the tile boundaries of JPEG2000 compressed images when wavelet transforms are used to perform lossy image compression. The boundary artifacts are due to error between the reconstructed and original images samples close to the boundary of a tile. To determine the cause of this error, we have investigated the error introduced by the lossy compression process at the boundary of the tile that has been transformed using CDF 9/7 wavelet transform.

Let us consider the following set of $n$ samples of an image:

$$
x[0], x[1], \ldots, x[n-2], x[n-1] .
$$


When single level wavelet transform is applied on this image, the following set of low pass and high pass subband samples are produced:

$$
\begin{aligned}
& y_{0}^{\prime}[0], y_{0}^{\prime}[2], \ldots, y_{0}^{\prime}[n-4], y_{0}^{\prime}[n-2] \text { and } \\
& y_{1}^{\prime}[1], y_{1}^{\prime}[3], \ldots, y_{1}^{\prime}[n-3], y_{1}^{\prime}[n-1] .
\end{aligned}
$$

These low pass and high pass subband samples are interleaved to get the following sequence

$$
y_{0}^{\prime}[0], y_{1}^{\prime}[1], \ldots, y_{0}^{\prime}[n-2], y_{1}^{\prime}[n-1] \text {. }
$$

From this, one can observer that for an even number of input samples, one end of the sample sequence is a low pass sample and other end is a high pass sample. In case of lossy compression, these subband samples are quantized in order to reduce the precision of the subband samples to aid in achieving higher compression. Quantization of subband samples is one of the main sources of information loss in the encoder. Quantization is performed by uniform scalar quantization with dead zone about the origin and is defined as:

$$
q_{b}[n]=\operatorname{sign}\left(y_{b}^{\prime}[n]\right)\left\lfloor\frac{\left|y_{b}^{\prime}[n]\right|}{\Delta_{b}}\right\rfloor
$$

where $y_{b}^{\prime}[n]$ is the sample of subband $b ; q_{b}[n]$ is the quantized sample; $\Delta_{b}$ is the quantization step size for subband $b$. The JPEG2000 standard supports separate quantization step size for each subband due to multiresolution feature supported by $D W T$. The quantization step size for a subband is calculated based on the dynamic range of the samples of the subband. The sign function is defined as:

$$
\operatorname{sign}(x)=\left\{\begin{array}{cl}
-1, & \text { if } x<0 \\
0, & \text { if } x=0 \\
1, & \text { if } x>0
\end{array}\right.
$$

After the quantization process, the subbands of a tile are divided into code blocks, which are compressed independently using Embedded Block Coding with Optimized Truncation (EBCOT) algorithm [3]. EBCOT defines the methodology for arranging the compressed output in the form of bit stream allowing the rate control with optimum quality. It is composed by two tiers. Tier- 1 generates the collection of encoded bits for each code block. Tier-2 reorganizes the output of tier1 by discarding some of the encoded bits in order to obtain the best possible quality for a given the compression ratio. On the decoder side, the steps of the encoder are executed in reverse order to decompress the images.

After the inverse quantization process on the decoder side, the reconstructed subband samples in subband $b$ are given by

$$
y_{b}^{\prime \prime}[n]=\operatorname{sign}\left(q_{b}[n]\right)\left(\left|\Delta_{b} \cdot q_{b}[n]\right|+\delta\right)
$$

where $\delta$ is usually set to $\Delta_{b} / 2$.

The reconstructed subband samples can then be expressed as

$$
y_{b}^{\prime \prime}[n]=y_{b}^{\prime}[n]+\varepsilon_{b}[n]
$$

where $\varepsilon_{b}[n]$ is the error, between the original subband sample and the reconstructed sample, caused by scalar quantization. It can be noted from (3) that errors are present in each of the reconstructed samples of decompressed images as the quantization process is irreversible. 


\subsection{Quality Parameters}

Peak signal-to-noise ratio (PSNR) between the original and reconstructed images is most commonly used objective quality measure. In this paper, $P S N R$ is used to measure the quality of the reconstructed images, which is a function of mean square error (MSE) and is defined by

$$
P S N R=10 \log _{10} \frac{\left(2^{B}-1\right)^{2}}{M S E}
$$

where $B$ is the bit depth of the image and MSE is defined as

$$
M S E=\frac{1}{M \times N} \sum_{i=0}^{M-1} \sum_{j=0}^{N-1}[\bar{I}(i, j)-I(i, j)]^{2}
$$

where $\bar{I}(i, j)$ is the pixel of the reconstructed image and $I(i, j)$ is the pixel of the original image and $M$ and $N$ represent the height and width of the image respectively.

\subsection{Error Analysis of Boundary Samples of Tiles of an Image}

Wei et al. [9] have shown that the error in high pass reconstructed boundary sample is higher than the low pass reconstructed boundary sample. On the lines of the work done by Wie et al.[9], we have also computed the errors in the boundary samples of the tiles of the image.

In order to analyze the errors in boundary samples of a tile, we have collected 10000 boundary samples of the tiles of the different original images, reconstructed images using JPEG2000 standard and reconstructed images with post-processing by proposed method. Figure 1 contains the results of this analysis. In this figure, sample 1 corresponds to the last but one reconstructed pixel of the $i^{\text {th }}$ tile, sample 2 corresponds to the last reconstructed pixel of the $i^{\text {th }}$ tile and sample 3 corresponds to first reconstructed pixel of $(i+1)^{\text {th }}$ tile. Here, it can be noted that sample 2 has a large error in comparison with sample 1 and sample 3, when the image is compressed using JPEG2000 standard. This error is significantly reduced when the proposed method is applied after JPEG2000 decompression and this leads to reduction in boundary artifacts in JPEG2000 reconstructed images. The reconstructed boundary samples that have been considered in this analysis are captured from thirty different images. These are standard images (including lena, moon, planes, house, cornfield etc.), considered by other researchers working in this field.

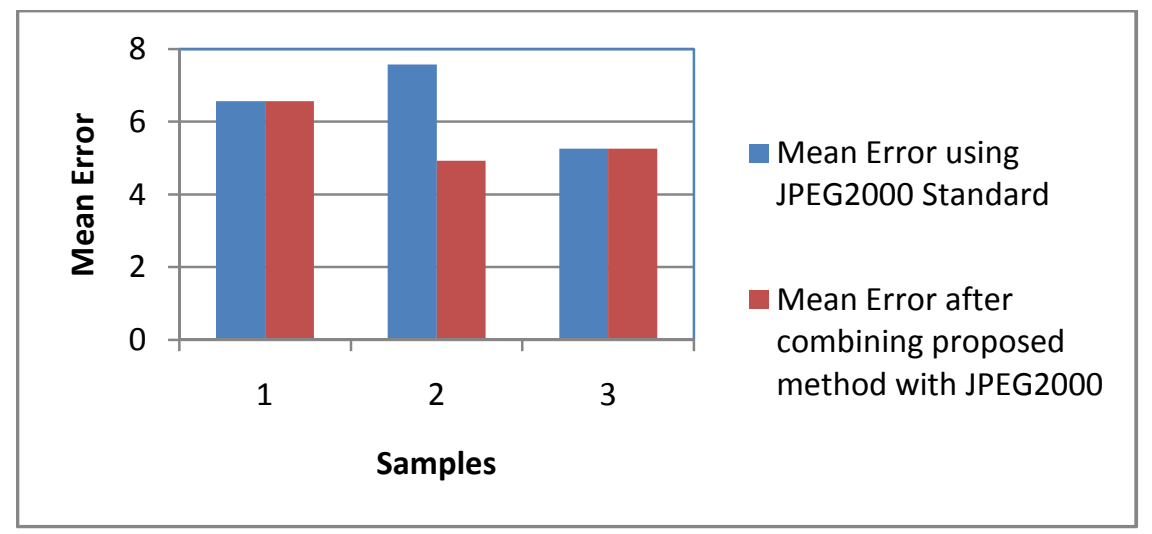

Figure 1: Mean of the pixel value differences of the boundary samples of image tiles. 
Now, let us analyze the MSE of the reconstructed samples at the tile boundaries of the decompressed images. The high pass boundary reconstructed samples have higher MSE than low pass boundary reconstructed samples as shown below in Figures 2. When these high pass boundary samples are post-processed using the proposed method, the MSE of these samples decreases, as shown below in Figure 3.
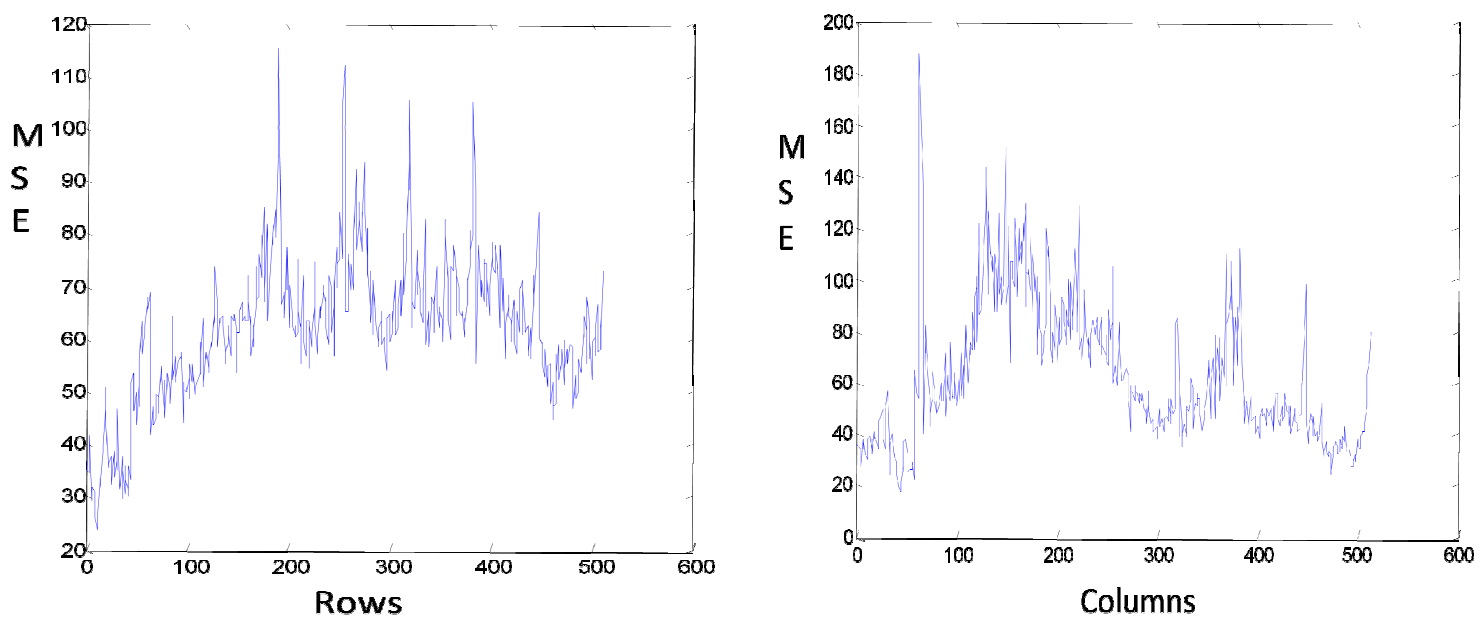

Figure 2: Average MSE for each row and column of the $512 \times 512$ Lena image after compression at a bit rate of 0.1 bits-per-pixel using JPEG2000 Part1 with tile size of $64 \times 64$.
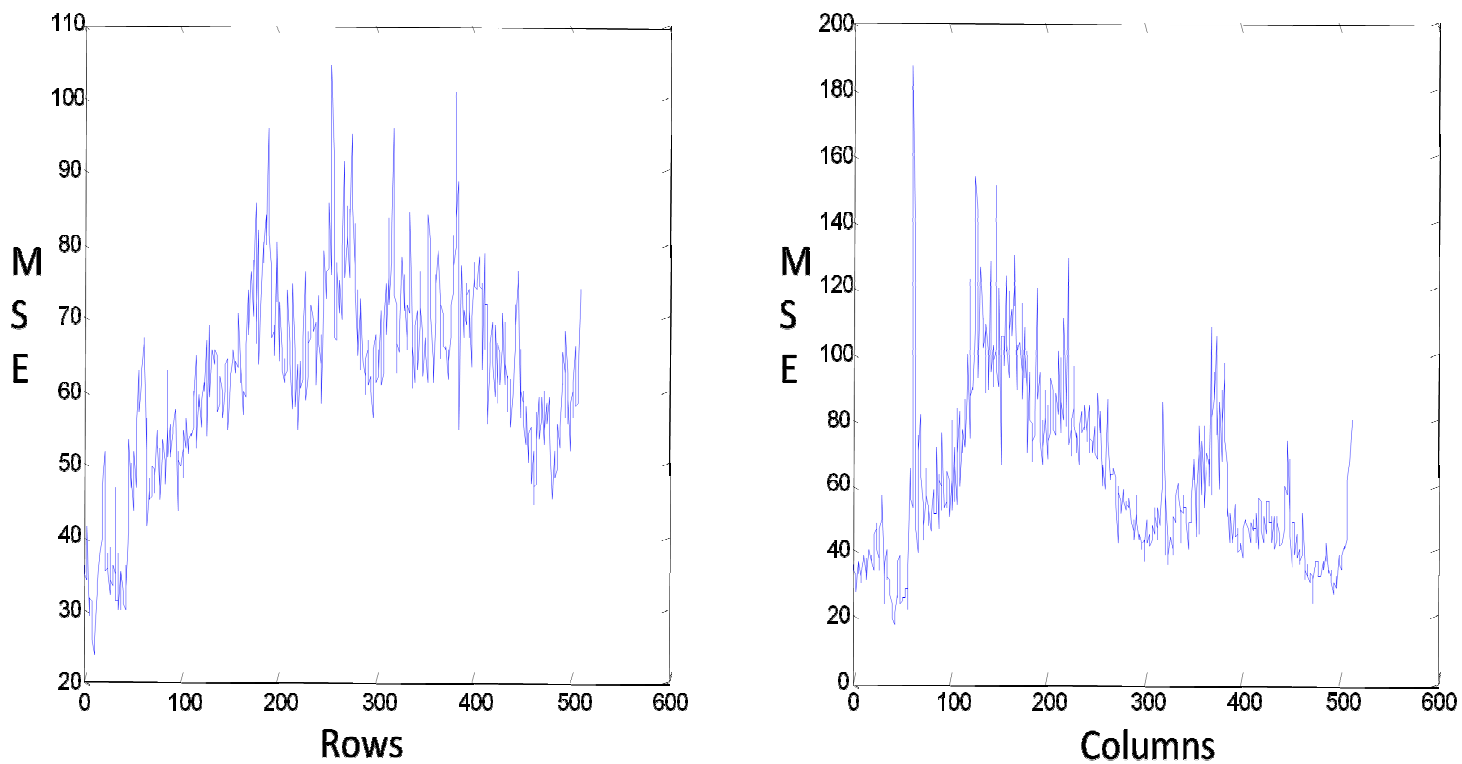

Figure 3: Average $M S E$ for each row and column of the $512 \times 512$ Lena image after post-processing the high pass boundary samples and compression at a bit rate of 0.1 bits-per-pixel using JPEG2000 Part1 with tile size of $64 \times 64$. 


\section{Tile Boundary Artifact Reduction}

To reduce tile boundary artifacts in a JPEG2000 reconstructed image, we have replaced the high pass reconstructed boundary samples by the average of left and right neighboring samples, when the vertical tile boundary is processed. While processing the horizontal tile boundaries, we have replaced the high pass reconstructed boundary samples by the average of top and bottom neighboring samples. By doing this, $M S E$ of the processed tile boundary samples is reduced and hence PSNR and visual quality of the reconstructed images are improved. The comparison of $P S N R$ values for thirty images, considered in this work, is given in Figure 4.

The results have again been obtained by taking a set of 30 images. These images are compressed at low bit rate using Kakadu software tool [13] with five level wavelet decomposition. The comparisons show that the result holds good for each of the images. From Figure 4, one can also observe that there is an improvement of 0.01 to $0.3 d \mathrm{~B}$ in the PSNR of the compressed images. Improvements in the visual quality of an image are shown in Figure 6. A reconstructed Lena image compressed at 0.1 bits-per-pixel, using JPEG2000 standard with a tile size of $64 \times 64$ is shown in Figure 5 and a reconstructed Lena image compressed at 0.1 bits-per-pixel, using $J P E G 2000$ with proposed post-processing with a tile size of $64 \times 64$ is shown in Figure 6.

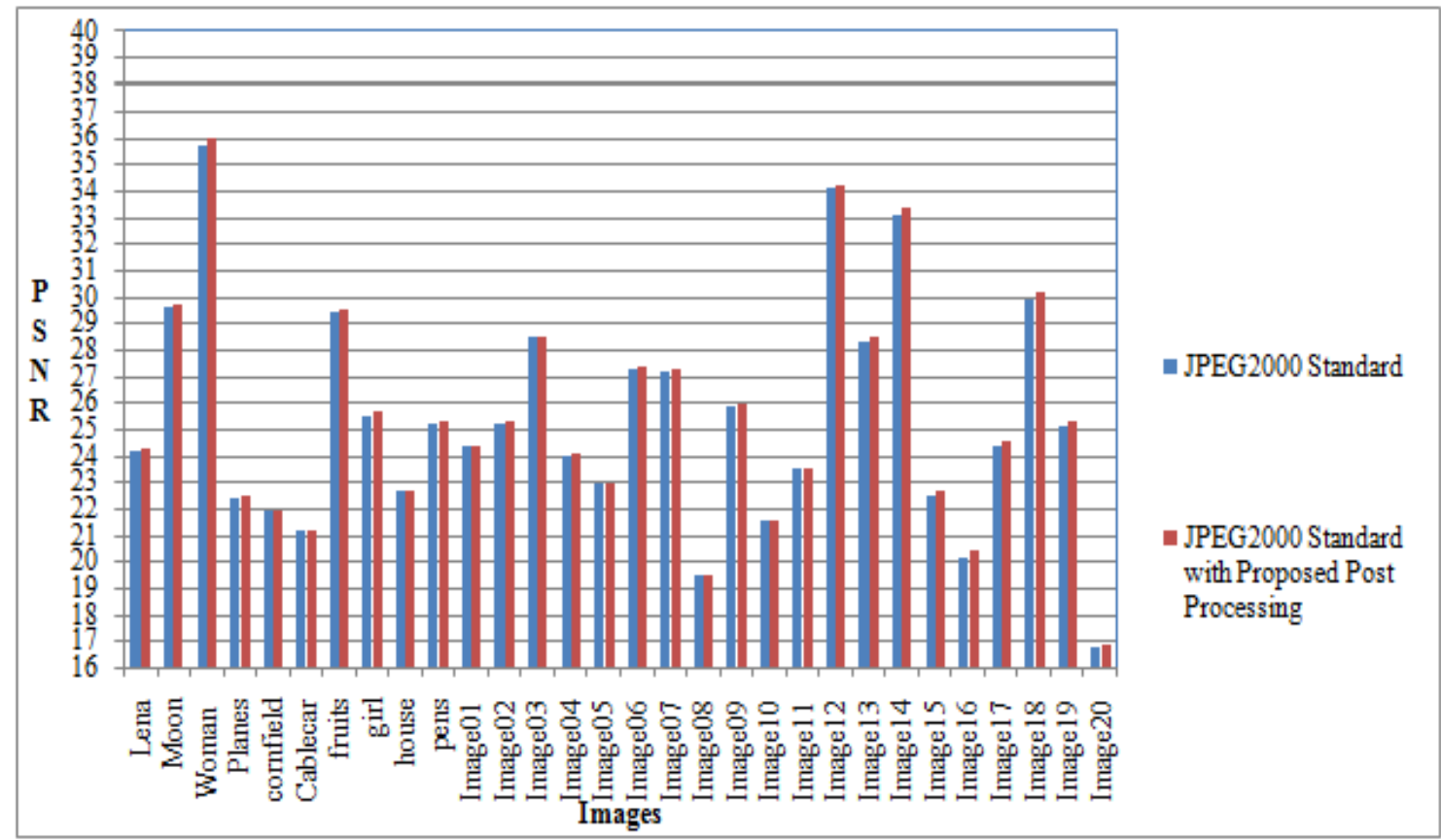

Figure 4: PSNR comparison of thirty images compressed at 0.1 bits-per-pixel using JPEG2000 standard and JPEG2000 standard with proposed post-processing method. 


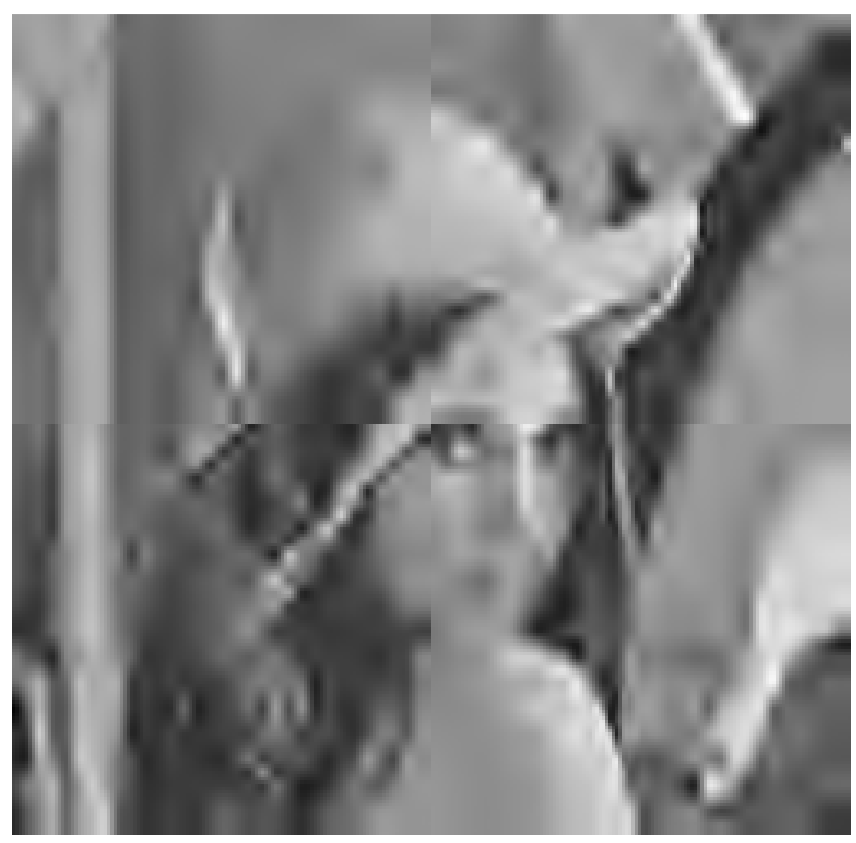

Figure 5: Decompressed $512 \times 512$ Lena image after compression at a bit rate of 0.1 bits-perpixel using JPEG2000 with a tile size of $64 \times 64$.

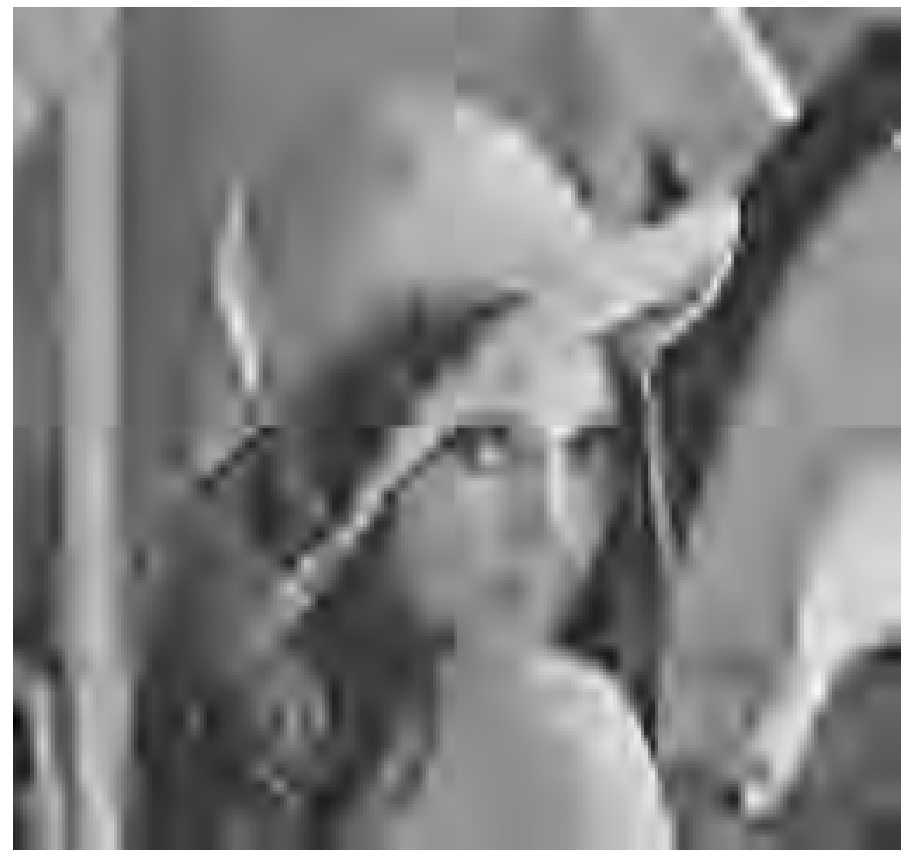

Figure 6: Decompressed $512 \times 512$ Lena image after compression at a bit rate of 0.1 bits-per-pixel using $J P E G 2000$ with proposed post-processing method and a tile size of $64 \times 64$. 


\section{Conclusion}

In this paper, a post-processing methodology has been implemented that can be applied along with JPEG200 standard for reducing boundary artifacts of tiled images. The methodology is motivated by the higher error in high pass reconstructed boundary samples than low pass boundary samples. It has been shown that when one uses proposed method, tile boundary artifacts are reduced in terms of increase in $P S N R$ value. This increase in $P S N R$ value varies from $0.01 d \mathrm{~B}$ to $0.3 d \mathrm{~B}$ for thirty different images considered in this work. The reductions in boundary artifacts have also been observed in visual quality of decompressed images.

\section{References}

[1] ISO/IEC 15444-1(2001) "Information Technology - JPEG2000 Image Coding System - Part 1: Core Coding System".

[2] Taubman D. S. and Marcellin M. W. (2002) JPEG2000: Image Compression Fundamentals, Standards and Practice, Boston, MA: Kluwer.

[3] Taubman D. S. (2000) "High Performance Scalable Image Compression with EBCOT", IEEE Transactions on Image Processing, Vol. 9, No. 7, pp 1158 - 1170.

[4] Varshney H., Hasan M., and Jain S. (2007) "Energy Efficient Novel Architectures for Lifting Based Discrete Wavelet Transform”, IET Image Processing, Vol. 1, No. 3, pp. $305-310$.

[5] Smorfa S., and Olivieri M. (2007) "HW-SW Optimization of JPEG2000 Wavelet Transform for Dedicated Multimedia Processor Architectures", IET Computers and Digital Techniques, Vol. 1, No. 2, pp. 137-143.

[6] Singara Singh, R. K. Sharma and M. K. Sharma (2009) "Use of Wavelet Transform Extension for Graphics Image Compression using JPEG2000 Framework", International Journal of Image Processing, Vol. 3, Issue 1, pp. 55-60.

[7] Berkner K. and Schwartz E. L. (2002), "Removal of tile artifacts using projection onto scaling functions for JPEG2000”, Proc. IEEE Int. Conf. on Image Processing, Rochester, NY, pp. 373-376.

[8] Kharitonenko I., Zhang X., and Twelves S. (2002), "A wavelet transform with point symmetric extension at tile boundaries", IEEE Transactions on Image Processing, Vol. 11, No. 12, pp. 13571364.

[9] Wei J., Pickering M., Frater M., Arnold J., Boman J., and Zeng W. (2005) "Tile Boundary Artifacts Reduction Using Odd Tile Size and the Low Pass First Convention", IEEE Transactions on Image Processing, Vol. 14, No. 8, pp. 1033- 1042.

[10] Liang J., Tu C., Tran T. D. (2003) "Optimal Pre- and Post-processing for JPEG2000 Tiling Artifacts Removal”, Conference on Information Sciences and Systems, The Johns Hopkins University, March.

[11] Qin X., Yan X., Yang C., and Ke Y. (2004) "Tiling Artifact Reduction for JPEG2000 Image at Low Bit Rate", IEEE International Conference on Multimedia and Expo, pp. 1419-1422.

[12] Hashimoto M., Matsuo K., and Kioke A. (2005) "JPEG2000 Encoding Method for Reducing Tiling Artifacts", IEICE Transaction on Information and Systems, Vol. E88 - D, No. 12, pp. 2839 - 2848. 
[13] Taubman D. S., Kakadu Software, www.kakadusoftware.com.

\section{Authors}

Singara Singh is currently working as Assistant Professor in School of Mathematics and Computer Applications, Thapar University, Patiala, India. He received his M. Sc. and M. Tech. degrees in 1998 and 2000, respectively. He is curren tly pursuing Ph. D. degree in image compression. His research interests include image processing, wireless networks and data security.

Dr. R. K. Sharma is Professor in School of Mathematics and Computer Applications, Thapar University, Patiala, India. He obtained his Ph. D. degree in 1993 from Indian Institute of Technology, Roorkee, India. He has published more than 30 papers in the area of pattern recognition, neural networks and ATM networks. His research interests include soft computing, neural networks, and statistical methods in NLP.

Dr. M. K. Sharma is Associate Professor in School of Mathem atics and Computer Applications, Thapar University, Patiala, India. He obtained his Ph. D. degree from Indian Institute of Technology, Roorkee, India. He has published several papers in the area of theoretical astrophysics and operations research. His research interests include theoretical astrophysics, multiobjective optimization and image processing.
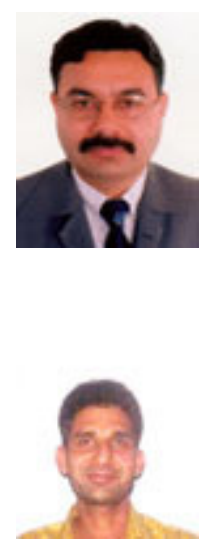